(C) 1982. The Genetical Society of Great Britain

\title{
ESTIMATION OF DOUBLE REDUCTION AND GENETIC PARAMETERS OF AUTOTETRAPLOIDS
}

\author{
G. C. C. TAl \\ Research Station, Agriculture Canada, Fredericton, New Brunswick, and Department \\ of Genetics, University of Birmingham, Birmingham
}

Received 22.ii.82

\section{SUMMARY}

\begin{abstract}
A method is proposed for estimating double reduction and genetic parameters in an additive-dominance model for autotetraploids. It uses a mating scheme involving a diploid hybrid and its tetraploid in a series of seven crosses. The diploid produces $2 n$ unreduced gametes by first and/or second division restitution during meiosis.
\end{abstract}

\section{INTRODUCTION}

Two major factors complicate the study of polygenic variation of autotetraploids. There are four alleles at a locus. This gives a complex pattern of genic interactions as compared to that of diploids. The situation can, however, be somewhat simplified by considering only two alleles per locus. A more troublesome problem is the phenomenon of double reduction, which was elucidated by Mather (1936). Most theoretical work, for example, Kempthorne (1955), Li (1957), Dessureaux (1959), Levings and Dudley (1963), Hill (1971) and Gallais (1974), consider a random mating population having chromosomal segregation. Killick (1971) and Tan (1976) investigated generations derived from a cross between two pure lines with allowance for double reduction. The complexity produced by the double reduction can be verified by the expressions for generation means in Killick's paper.

The discovery by Peloquin and his colleagues (Mendiburu, Peloquin and Mok, 1974) that certain interspecific diploids in potato produce $2 n$ unreduced gametes provides a new tool for genetic studies of autotetraploids. The $2 n$ gametes are produced by either first (FDR) or second (SDR) division restitution during meiosis (Mendiburu and Peloquin, 1979). As exemplified by many experiments (e.g., Hanneman and Peloquin, 1969; Mendiburu and Peloquin, 1971; De Jong and Tai, 1977; McHale and Laurer, 1981), there seems no difficulty in obtaining tetraploid progenies from diploid-diploid $(2 x \times 2 x)$ and diploid-tetraploid $(2 x \times 4 x)$ matings.

In the present paper a procedure is proposed for estimating the double reduction coefficient and genetic parameters in an "additive-dominance" model for autotetraploids. It is based on a mating system involving a series of crosses between diploids and tetraploids.

\section{BASIC CONSIDERATIONS}

The additive-dominance model considers two alleles per locus with the five possible tetraploid genotypes taking the following values: 


$\begin{array}{llll}A A A A & \text { or } & A_{4} & m+d \\ A A A a & \text { or } & A_{3} a & m+h_{3} \\ A A a a & \text { or } & A_{2} a_{2} & m+h_{2} \\ \text { Aaaa } & \text { or } & A a_{3} & m+h_{1} \\ \text { aaaa } & \text { or } & a_{4} & m-d .\end{array}$

This is the model developed by Mather and Jinks (1971) and Killick (1971).

A feature of the proposed procedure is the use of a diploid genotype $A a$ and its tetraploid $A_{2} a_{2}$ in a series of seven crosses. The diploid produces unreduced $2 n$ gametes by either FDR or SDR during meiosis. We shall, however, concentrate on the FDR diploid since the principle of using the SDR diploid is exactly the same. It is necessary to know the gametic output of the above five tetraploids and the FDR diploid, and the frequencies of progeny genotypes when they are crossed with two parents $A a$ (FDR) and $A_{2} a_{2}$, before we proceed with the mating scheme. Table 1 gives the gametic output of the tetraploids (Fisher and Mather, 1943) and FDR diploid (Mendiburu and Peloquin, 1979). Different sizes are allowed for the frequencies of double reduction $(\alpha)$ in the tetraploid and single exchange tetrads $(\beta)$ in the diploid genotypes.

TABLE 1

Gametic output of five autotetraploids and a FDR diploid

\begin{tabular}{ccccc} 
& \multicolumn{3}{c}{ Gametes } & \\
Parental genotype & $\overbrace{A A}$ & $A a$ & $a a$ & Divisor \\
$A_{4}$ & 1 & - & - & 1 \\
$A_{3} a$ & $2+\alpha$ & $2-2 \alpha$ & $\alpha$ & 4 \\
$A_{2} a_{2}$ & $1+2 \alpha$ & $4-4 \alpha$ & $1+2 \alpha$ & 6 \\
$A a_{3}$ & $\alpha$ & $2-2 \alpha$ & $2+\alpha$ & 4 \\
$a_{4}$ & $\bar{\beta}$ & $4-2 \beta$ & $\beta$ & 1 \\
$A a($ FDR $)$ & & &
\end{tabular}

There are again five possible genotypes in the progenies when any one of the five tetraploids $\left(A_{4}, A_{3} a, A_{2} a_{2}, A a_{3}\right.$ and $\left.a_{4}\right)$ or FDR diploid $(A a)$ is crossed with $A a$ or $A_{2} a_{2}$. The frequencies of five genotypes in the progenies of $A a \times A_{3} a$ and $A_{2} a_{2} \times A_{3} a$, for example, are given in table 2. The expressions of the frequencies, as shown in the second and third columns in table 2, appear to be complicated. The difference between pairs of frequencies for each of the progeny genotypes from the two crosses, which are given in the fourth column in table 2 , assume rather simpler forms. This is generally true for the other four tetraploids and the FDR diploid crossed with $A a$ and $A_{2} a_{2}$. The results for all the differences are shown in table 3.

The differences between mean genotypic values of the crosses $\mathrm{Aa} \times \mathrm{A}_{3} a$ and $A_{2} a_{2} \times A_{3} a$ can be easily obtained by using the results in table 2 , i.e.,

$$
\begin{aligned}
& \overline{\left(A a \times A_{3} a\right)}-\overline{\left(A_{2} a_{2} \times A_{3} a\right)} \\
& =\gamma\left[-\left(\frac{2+\alpha}{4}\right) d+\left(\frac{2+4 \alpha}{4}\right) h_{3}+\left(\frac{2-6 \alpha}{4}\right) h_{2}-\left(\frac{2-4 \alpha}{4}\right) h_{1}-\left(\frac{\alpha}{4}\right)(-d)\right] \\
& =\frac{\gamma}{2}\left[-d+(1+2 \alpha) h_{3}+(1-3 \alpha) h_{2}-(1-2 \alpha) h_{1}\right]
\end{aligned}
$$

in which $\gamma=(2+4 \alpha-3 \beta) / 12$. 


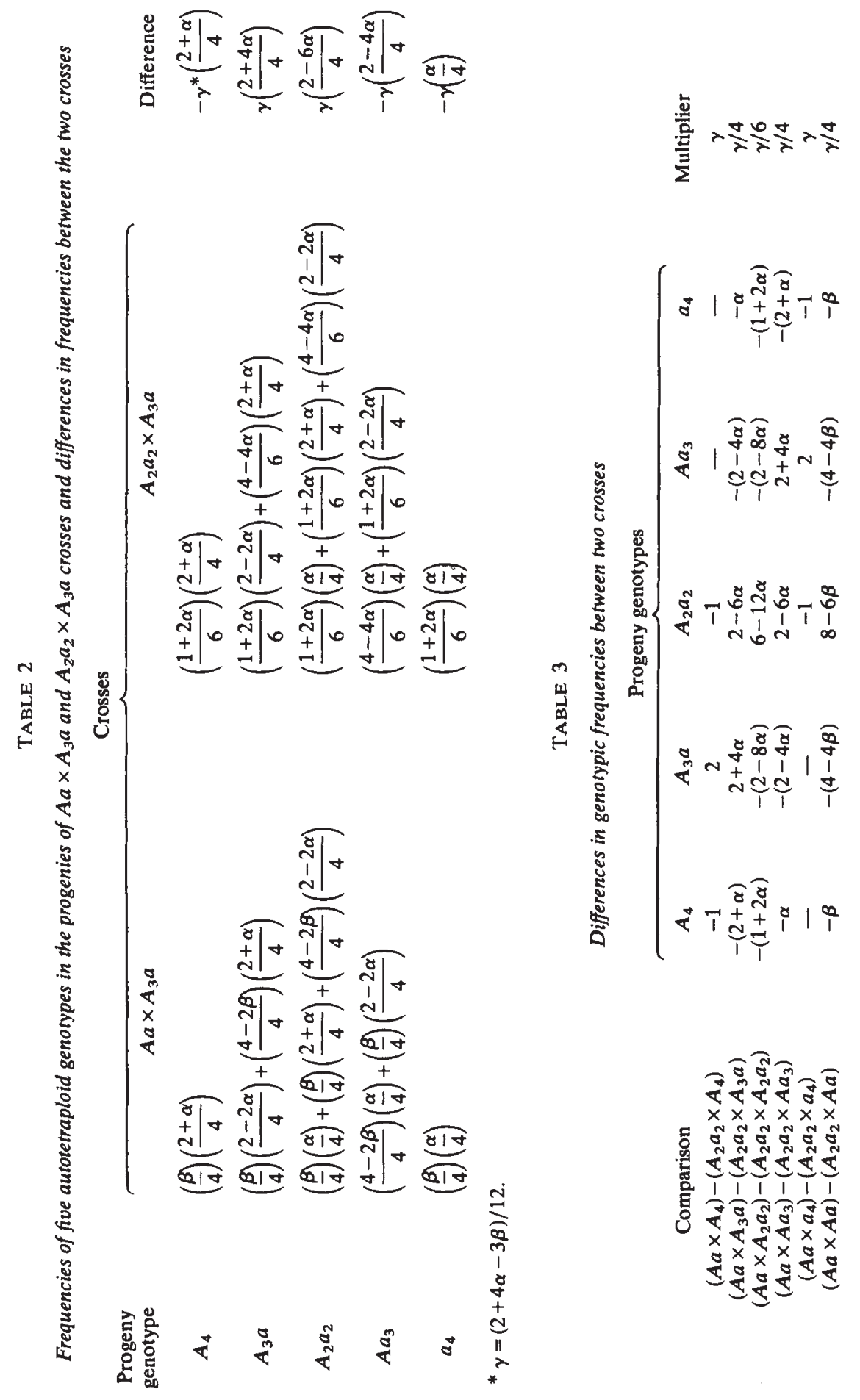


TABLE 4

Differences between genotypic means of two crosses

Comparison

$\left(A a \times A_{4}\right)-\left(A_{2} a_{2} \times A_{4}\right)$

$\left(A a \times A_{3} a\right)-\left(A_{2} a_{2} \times A_{3} a\right)$

$\left(A a \times A_{2} a_{2}\right)-\left(A_{2} a_{2} \times A_{2} a_{2}\right)$

$\left(A a \times A a_{3}\right)-\left(A_{2} a_{2} \times A a_{3}\right)$

$\left(A a \times a_{4}\right)-\left(A_{2} a_{2} \times a_{4}\right)$

$(A a \times A a)-\left(A_{2} a_{2} \times A a\right)$
Difference

Symbol

$$
\gamma\left[-d+2 h_{3}-h_{2}\right]
$$

$d_{1}^{\prime}$

$\frac{\gamma}{2}\left[-d+(1+2 \alpha) h_{3}+(1-3 \alpha) h_{2}-(1-2 \alpha) h_{1}\right]$

$h_{3}^{\prime}$

$\frac{\gamma}{3}\left[-(1-4 \alpha)\left(h_{3}+h_{1}\right)+(3-6 \alpha) h_{2}\right]$

$h_{2}^{\prime}$

$\frac{\gamma}{2}\left[d-(1-2 \alpha) h_{3}+(1-3 \alpha) h_{2}+(1+2 \alpha) h_{1}\right]$

$h_{1}^{\prime}$

$\gamma\left[d-h_{2}+2 h_{1}\right]$

$d_{2}^{\prime}$

$\frac{\gamma}{2}\left[-(2-2 \beta)\left(h_{3}+h_{1}\right)+(4-3 \beta) h_{2}\right]$

Table 4 gives the differences between pairs of mean genotypic values of progenies obtained from crossing $A a$ and $A_{2} a_{2}$ with each of the five tetraploids and the FDR diploid. The expressions of all six differences, again, are relatively simple.

\section{Method of estimation}

We may now propose a mating scheme using $A a$ and $A_{2} a_{2}$ as parents for estimating the frequencies of double reduction $(\alpha)$ and single exchange tetrads $(\beta)$ and genetic parameters $\left(m, h_{3}, h_{2}\right.$ and $\left.h_{1}\right)$. We assume that $A a$ produces FDR $2 n$ gametes and there is no difficulty in extracting tetraploid progenies from such crosses as $A a \times A a, A a \times A_{2} a_{2}$, etc. Let $P_{1}=A a$ and $P_{2}=A_{2} a_{2}$. A series of seven crosses are made as follows:

\section{Type of crosses}

$$
\begin{aligned}
& 2 x \times 2 x \\
& 2 x \times 4 x \\
& 4 x \times 4 x \\
& 2 x \times 4 x \\
& 4 x \times 4 x \\
& 2 x \times 4 x \\
& 4 x \times 4 x
\end{aligned}
$$

Mating scheme

$$
A a \times A a
$$$$
\mathrm{Aa} \times \mathrm{A}_{2} \mathrm{a}_{2}
$$$$
A_{2} a_{2} \times A_{2} a_{2}
$$$$
\mathrm{Aa} \times\left(\mathrm{Aa} \times \mathrm{A}_{2} \mathrm{a}_{2}\right)
$$$$
A_{2} a_{2} \times\left(A a \times A_{2} a_{2}\right)
$$$$
A a \times\left(A_{2} a_{2} \times A_{2} a_{2}\right)
$$$$
A_{2} a_{2} \times\left(A_{2} a_{2} \times A_{2} a_{2}\right)
$$

Symbols

$$
\begin{gathered}
F_{11} \\
F_{12} \\
F_{22} \\
B_{1(12)} \\
B_{2(12)} \\
B_{1(22)} \\
B_{2(22)} .
\end{gathered}
$$

The mean values of the seven crosses, together with that of $P_{2}$, are combined to simplify the procedure of estimation. Using results in table 4,

$$
\begin{aligned}
\bar{F}_{12}-\bar{F}_{22} & =\frac{\gamma}{3}\left[-(1-4 \alpha)\left(h_{3}+h_{1}\right)+(3-6 \alpha) h_{2}\right] \\
\bar{F}_{11}+\bar{F}_{22}-2 \bar{F}_{12} & =2 \gamma^{2}\left[-2\left(h_{3}+h_{1}\right)+3 h_{2}\right] .
\end{aligned}
$$

Also,

$$
\begin{aligned}
\left(\bar{B}_{1(12)}\right. & \left.-\bar{B}_{2(12)}\right)-\left(\bar{B}_{1(22)}-\bar{B}_{2(22)}\right) \\
& =\frac{\gamma}{3}\left[-(1+2 \alpha)\left(d_{1}^{\prime}+d_{2}^{\prime}\right)-(1-4 \alpha)\left(h_{3}^{\prime}+h_{1}^{\prime}\right)+(3-6 \alpha) h_{2}^{\prime}\right] \\
& =\frac{\gamma^{2}}{3}\left[-(2-2 \alpha)\left(h_{3}+h_{1}\right)+(3-3 \alpha) h_{2}\right]
\end{aligned}
$$


where the compositions of $d_{1}^{\prime}, d_{2}^{\prime}, h_{3}^{\prime}, h_{2}^{\prime}$ and $h_{1}^{\prime}$ are as given in table 4 . The derivation of (3) is more complex. Using information from tables 1 and 4, a step-by-step illustration of the contribution of the genotype $\boldsymbol{A}_{3} a$ to (3) is given as follows:

(i) The $\left(A a \times A_{2} a_{2}\right)$ cross produces the progeny $A_{3} a$ with the frequency

$$
\left[\left(\frac{\beta}{4}\right)\left(\frac{4-4 \alpha}{6}\right)+\left(\frac{2-\beta}{2}\right)\left(\frac{1+2 \alpha}{6}\right)\right]
$$

(ii) The $\left(A_{2} a_{2} \times A_{2} a_{2}\right)$ cross produces the progeny $A_{3} a$ with the frequency

$$
\left[2\left(\frac{4-4 \alpha}{6}\right)\left(\frac{1+2 \alpha}{6}\right)\right]
$$

(iii) The progeny $A_{3} a$ is crossed with $P_{1}(A a)$ and $P_{2}\left(A_{2} a_{2}\right)$. The difference between mean values of the crosses $\left(A a \times A_{3} a\right)$ and $\left(A_{2} a_{2} \times A_{3} a\right)$ is $h_{3}^{\prime}$.

(iv) The contribution of $A_{3} a$ to the difference $\left(\bar{B}_{1(12)}-\bar{B}_{2(12)}\right)$ is then

$$
\left[\left(\frac{\beta}{4}\right)\left(\frac{4-4 \alpha}{6}\right)+\left(\frac{2-\beta}{2}\right)\left(\frac{1+2 \alpha}{6}\right)\right] h_{3}^{\prime}
$$

whereas that to the difference $\left(B_{1(22)}-B_{2(22)}\right)$ is

$$
\left[2\left(\frac{4-4 \alpha}{6}\right)\left(\frac{1+2 \alpha}{6}\right)\right] h_{3}^{\prime}
$$

(v) Therefore, the contribution of $A_{3} a$ to the difference in (3) is

$$
\left[\left(\frac{\beta}{4}\right)\left(\frac{4-4 \alpha}{6}\right)+\left(\frac{2-\beta}{2}\right)\left(\frac{1+2 \alpha}{6}\right)-2\left(\frac{4-4 \alpha}{6}\right)\left(\frac{1+2 \alpha}{6}\right)\right] h_{3}^{\prime}=-\frac{\gamma}{3}(1-4 \alpha) h_{3}^{\prime} \text {. }
$$

The contributions of progeny genotypes $A_{4}, A_{2} a_{2}, A a_{3}$ and $a_{4}$ are similarly worked out as $-(\gamma / 6)(1+2 \alpha) d_{1}^{\prime}, \gamma(1-2 \alpha) h_{2}^{\prime},-(\gamma / 3)(1-4 \alpha) h_{1}^{\prime}$, and $-(\gamma / 6)(1+2 \alpha) d_{2}^{\prime}$, respectively. Adding them together we obtain (3).

Let

$$
\begin{aligned}
& w_{1}=\bar{F}_{12}-\bar{F}_{22} \\
& w_{2}=\bar{F}_{11}+\bar{F}_{22}-2 \bar{F}_{12} \\
& w_{3}=\bar{B}_{1(12)}-\bar{B}_{2(12)}-\bar{B}_{1(22)}+\bar{B}_{2(22)} .
\end{aligned}
$$

Substituting these expressions in (1), (2) and (3),

$$
\begin{aligned}
-(1-4 \alpha)\left(h_{3}+h_{1}\right)+(3-6 \alpha) h_{2} & =\frac{3 w_{1}}{\gamma} \\
-2\left(h_{3}+h_{1}\right)+\quad 3 h_{2} & =\frac{w_{2}}{2 \gamma^{2}} \\
-(2-2 \alpha)\left(h_{3}+h_{1}\right)+(3-3 \alpha) h_{2} & =\frac{3 w_{3}}{\gamma^{2}}
\end{aligned}
$$


$\alpha$ can be solved from (5) and (6), i.e.,

$$
\alpha=1-\frac{6 w_{3}}{w_{2}} \text {. }
$$

Also, $h_{2}$ and $\left(h_{3}+h_{1}\right)$ can be expressed in terms of $\gamma$ :

$$
\begin{aligned}
h_{2} & =\frac{2 w_{1}}{\gamma}+(4 \alpha-1) \frac{w_{2}}{6 \gamma^{2}} \\
\left(h_{3}+h_{1}\right) & =\frac{3 w_{1}}{\gamma}+(2 \alpha-1) \frac{w_{2}}{2 \gamma^{2}} .
\end{aligned}
$$

Solutions of $\left(h_{3}+h_{1}\right), h_{2}, \alpha$ and $m$ are obtained by using the difference between $\bar{F}_{22}$ and $\bar{P}_{2}$, i.e.,

$$
\bar{F}_{22}-\bar{P}_{2}=\frac{1}{36}\left\{2(1+2 \alpha)(4-4 \alpha)\left(h_{3}+h_{1}\right)+\left[2(1+2 \alpha)^{2}+(4-4 \alpha)^{2}\right] h_{2}\right\}-h_{2} \text {. }
$$

Let $w_{4}=\bar{F}_{22}-\bar{P}_{2}$ and express $(7)$ in terms of $\gamma$,

$$
\gamma^{2}+\left(\frac{w_{1} w_{2}-4 w_{1} w_{3}}{w_{2} w_{4}}\right) \gamma+\frac{\left(w_{2}-4 w_{3}\right)^{2}}{4 w_{2} w_{4}}=0
$$

We can then solve this equation for $\gamma$. This leads to estimates of $h_{2},\left(h_{3}+h_{1}\right)$, $\beta$ and $m$. The componcnt $d$ cannot be estimated and $h_{3}$ and $h_{1}$ cannot be separated.

The estimation procedure involving SDR diploid $(A a)$ is exactly the same as the one described above. The only difference is the structure of $\gamma$ which is $(2 \alpha+3 \beta-2) / 6$. This is derived from the gametic output of the SDR diploid $A a$, i.e., $A A: A a: a a=(1-\beta) / 2: \beta:(1-\beta) / 2$ (Mendiburu and Peloquin, 1979).

\section{DisCUSSION}

The estimation procedure depends on the simplicity of the differences between specific crosses (table 4). It is interesting to note the development bears a close resemblance to the method used by Kearsey and Jinks (1968) for the triple test cross.

In potatoes, the diploid hybrids are often obtained by crossing a haploid $(2 n=24)$ extracted from the cultivated species Solanum tuberosum $(2 n=$ 48 ) with a diploid species such as S. phureja $(2 n=24)$ (Hanneman and Peloquin, 1969). All the various parameters that can be estimated by the present method apply to the specific series of crosses derived from a diploid hybrid, rather than to either of the original species. The analytical method is in fact a useful tool for analysing variation within an autotetraploid species so long as the extracted haploids from the tetraploids will themselves produce $2 n$ unreduced gametes.

Because of the symmetrical distributions of the gametic frequencies in all seven crosses in the proposed mating scheme, $d$ is inestimable. Also, $h_{3}$ and $h_{1}$ can only be jointly estimated. A possibility for estimating $d, h_{3}$ and $h_{1}$ separately is to extract a homozygous haploid from a tetraploid pure line $\left(e . g ., A_{4}\right)$. The haploid $(A A)$ is then crossed with another extracted haploid genotype or diploid species to produce the diploid parent $\left(P_{1}\right)$ used 
in the mating scheme. In this case, data from the tetraploid pure line itself can be used for obtaining an estimate of $d$. It can also be used to cross with $P_{1}$ (i.e., $A a \times A_{4}$ ) and $P_{2}$ (i.e., $A_{2} a_{2} \times A_{4}$ ). Hybrids produced by the two extra crosses will give an estimate of $d_{1}^{\prime}$ (see table 4) which in turn provides information for separate estimates of $h_{3}$ and $h_{1}$.

The method works when the $2 n$ gametes are produced by either the FDR or SDR mechanisms. It is in fact also valid when the pool of $2 n$ gametes are contributed by both FDR and SDR. The gametic output of the diploid hybrid in this generalized situation is

$$
A A: A a: a a=\frac{2-2(\beta+\delta)+3 \beta \delta}{4}: \frac{2(\beta+\delta)-3 \beta \delta}{2}: \frac{2-2(\beta+\delta)+3 \beta \delta}{4}
$$

where $0 \leqq \delta \leqq 1$ (Mendiburu and Peloquin, 1979). The variable in (1)-(7) is then expressed as

$$
\gamma=\frac{4+6(\beta+\delta)-9 \beta \delta-4}{12} .
$$

The gametic ratios and $\gamma$ of FDR and SDR are derived by putting $\delta=1$ and $\delta=0$ in the above formulae respectively. It is clear that the parameter $\beta$ cannot be estimated when the mechanism of $2 n$ gamete production is neither FDR nor SDR but a mixture of both. The other three parameters $\left(\alpha, h_{2}\right.$ and $\left.h_{3}+h_{1}\right)$ can be estimated without difficulty. The parameter $\beta$, however, is less important than others in the study of tetrasomic inheritance.

The estimates of $h_{2}$ and $\left(h_{3}+h_{1}\right)$ in a polygenic system represent a summation over all heterozygous loci affecting the character under study. For the estimate of the summed effect of $h_{2}$ (or $h_{3}+h_{1}$ ) to be unbiased, it must be assumed that the size of an individual $h_{2}$ is not associated with those of $\alpha$ over all loci. In other words, the magnitude of a genic effect has nothing to do with the gene's location on the chromosome. In general, such an assumption appears genetically reasonable. The estimates of $\alpha$ and $\beta$ then represent the average values over all loci.

Acknowledgments.-I wish to express my sincere thanks to Professor J. L. Jinks for his guidance and helpful discussions during the study, and Dr Peter Caligari for his suggestions to improve the paper. The support of Agriculture Canada for my educational leave at the Department of Genetics is gratefully acknowledged.

\section{REFERENCES}

DE JONG, H., AND TAI, G. C. C. 1977. Analysis of tetraploid-diploid hybrids in cultivated potatoes. Potato Res., 20, 111-121.

DESSUREAUX, L. 1959. Introduction to the autotetraphid diallel. Can. J. Genet. Cytol., 1, 94-101.

FISHER, R. A., AND MATHER, K. 1943. The inheritance of style length in Lythrum salicaria. Ann. Eugenics, 12, 1-23.

GALlAIS, A. 1974. Covariances between arbitrary relatives in autotetraploids with panmictic disequilibrium. Genetics, 76, 587-600.

HANNEMAN, R. E., AND PELOQUIN, S. J. 1969. Use of Pheureja and haploids to enhance the yield of cultivated tetraploid potatoes. Am. Potato J., 46, 436.

HILL, R. R. JR. 1971. Selection in autotetraploids. Theor. Appl. Genet., 41, 181-186.

KEARSEY. M. J., AND JINKS, J. L. 1968. A general method of detecting additive, dominance and epistatic variation for metrical traits. Heredity, 23, 403-409.

KEMPTHORNE, O. 1955. The correlation between relatives in a simple autotetraploid population. Genetics, 40, 168-174. 
KILLICK, R. J. 1971. The biometrical genetics of autotetraploids. 1. Generations derived from a cross between two pure lines. Heredity, 27, 331-346.

LEVINGS, C. S., III, AND DUDLEY, J. W. 1963 . Evaluation of certain mating designs for estimation of genetic variance in autotetraploid alfalfa. Crop Sci., 3, 532-535.

LI, C. C. 1957. The genetic variance of autotetraploids with two alleles. Genetics, 42, 583-592. MATHER, K. 1936. Segregation and linkage in autotetraploids. J. Genetics, 32, 287-314.

MATHER, K., AND JINKS, J. L. 1971. Biometrical Genetics. Second edition. Chapman Hall, London.

McHALE, N. A., AND LAUER, F. I. 1981. Breeding value of $2 n$ pollen from diploid hybrids and Phureja in $4 X-2 X$ crosses in potatoes. Am. Potato J., 58, 365-374.

MENDIBURU, A. O., AND PELOQUIN, S. J. 1971. High yielding tetraploids from $4 X-2 X$ and $2 X-2 X$ matings. Am. Potato J., 48, 300-301.

MENDIBURU, A. O., AND PELOQUIN, S. J. 1979 . Gene-centromere mapping by $4 X-2 X$ matings in potatoes. Theor. Appl. Genet., 54, 177-180.

MENDIBURU, A. O., PELOQUIN, S. J., AND MOK, D. W. S. 1974. Potato breeding with haploids and $2 n$ gametes. In K. J. Kasha (ed.) Haploids in higher plants. Advances and potential. Univ. of Guelph, Ont. pp. 249-259.

TAN, W. Y. 1976. On the biometrical genetics of autotetraploid from a cross between two pure lines. Bot. Bull. Academia Sinica, 17, 1-17. 